\title{
Multichannel Poly-Si Thin-Film Transistors Prepared by Excimer Laser Annealing With Channel Width Comparable or Smaller Than the Grain Size
}

\author{
Po-Chuan Yang, Ping-Sheng Kuo, and Si-Chen Lee, Fellow, IEEE
}

\begin{abstract}
This paper presents results on low-temperature $\left(<500{ }^{\circ} \mathrm{C}\right)$ multichannel poly-Si thin-film transistors (TFTs) prepared by KrF excimer laser annealing with a channel width that is comparable to or smaller than the poly-Si grain size. The crosssectional scanning electron microscope is used to measure the effective channel width, and TCAD software is used to simulate the electron density distribution in the channel region. It is found that the TFTs with ten 40-nm-wide multichannels have superior electrical characteristics, including a higher on/off current ratio $\left(>10^{7}\right)$, lower leakage current $\left(8.8 \times 10^{-14} \mathrm{~A}\right)$, less grain boundary defects density, and a better subthreshold swing $(0.45 \mathrm{~V} / \mathrm{dec})$.
\end{abstract}

Index Terms-Polycrystalline silicon, thin-film transistor (TFT).

\section{INTRODUCTION}

$\mathbf{R}$ ECENTLY, there has been much interest in lowtemperature poly-Si (LTPS) thin-film transistors (TFTs) technology for active matrix liquid crystal display [1] and active matrix organic light-emitting diode [2] due to their high carrier mobility. It is expected that all functional components made of poly-Si TFTs can be integrated on a substrate for systemon-chip [3] and system-on-panel applications. However, owing to the existence of grain boundary defects in the channel region, the OFF-state leakage current and threshold voltage $\left(V_{\mathrm{th}}\right)$ of the poly-Si TFTs are relatively high, as compared with those of hydrogenated amorphous silicon (a-Si:H) TFTs [4].

Hence, it is very essential to minimize the grain boundary defects in order to improve the electrical characteristics of polySi TFTs. It has been reported that the poly-Si TFTs with several multichannels can effectively reduce the grain boundary defects [5]-[7]. Moreover, the TFT characteristics are dramatically improved with a reduced channel width, particularly when the channel width is close to the grain size [8], [9]. Therefore, the relation between the grain size and the channel width is a critical issue when the device dimension is scaled down.

This paper will present the results of poly-Si TFTs when the channel width is smaller than the grain size. The amorphous sil-

Manuscript received March 20, 2008; revised April 21, 2008. This work was supported by the National Science Council of the Republic of China under Contract NSC 95-2221-E-002-298. The review of this paper was arranged by Editor M. J. Kumar.

P.-C. Yang and P.-S. Kuo are with the Graduate Institute of Electronics Engineering, National Taiwan University, Taipei 106, Taiwan, R.O.C.

S.-C. Lee is with the Graduate Institute of Electronics Engineering and with the Department of Electrical Engineering, National Taiwan University, Taipei 106, Taiwan, R.O.C. (e-mail: sclee@ @cc.ee.ntu.edu.tw).

Color versions of one or more of the figures in this paper are available online at http://ieeexplore.ieee.org.

Digital Object Identifier 10.1109/TED.2008.925288

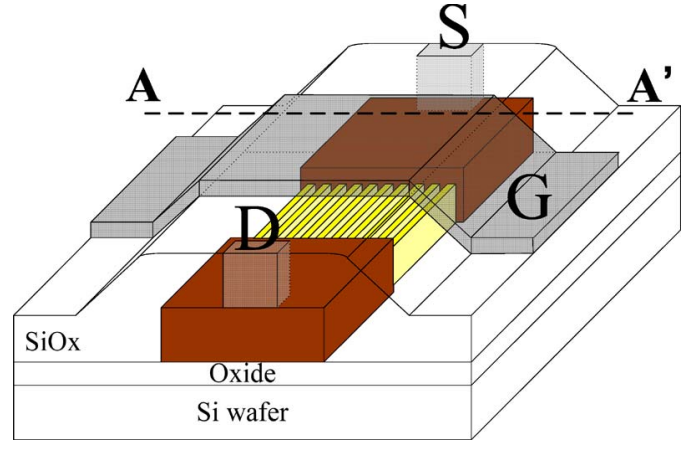

Fig. 1. Schematic diagram showing the device structure of a low-temperature ten 40-nm-wide channel poly-Si TFT prepared by $\mathrm{KrF}$ excimer laser annealing.

icon deposited by plasma-enhanced chemical vapor deposition (PECVD) is first annealed at $500{ }^{\circ} \mathrm{C}$ for $10 \mathrm{~min}$ in order to drive out the hydrogen atoms and then irradiated by $\mathrm{KrF}$ excimer laser in order to form a polycrystalline grain with an average size of $\sim 200 \mathrm{~nm}$. Single-, double-, and ten-channel poly-Si TFTs, with a channel width that is comparable to (400 and $200 \mathrm{~nm})$ or smaller $(40 \mathrm{~nm})$ than the grain size $(200 \mathrm{~nm})$, are fabricated, and their electrical characteristics are investigated. Unlike the double gate FinFET with a 17-nm-thick gate insulator [10], the 100-nm-thick PECVD $\mathrm{SiO}_{x}$ is used here. Hence, the cross-sectional scanning electron microscope (SEM) is used to measure the total effective channel width, and the TCAD simulator is used to simulate the electron density distribution in the channel region. Finally, the performance of TFTs, including its electrical characteristics, $V_{\mathrm{th}}, I_{\mathrm{min}}$, on/off current ratio, mobility, and grain boundary defects, are compared.

\section{EXPERIMENTS}

Fig. 1 shows the schematic diagram of this process. First, 50-nm-thick intrinsic a-Si:H and 20-nm-thick n-type phosphorus-doped a-Si:H layers are deposited by PECVD on oxidized Si substrate. During deposition, the substrate temperature, RF power density, and chamber pressure are fixed at $250{ }^{\circ} \mathrm{C}, 0.11 \mathrm{~W} / \mathrm{cm}^{2}$, and 0.45 torr, respectively. As for the gas flow rate, the undoped a-Si:H layer is $3\left(\mathrm{H}_{2}\right)$ and $3\left(\mathrm{SiH}_{4}\right)$ sccm, and the n-type doped a-Si:H layer is $2\left(\mathrm{H}_{2}\right), 3\left(\mathrm{SiH}_{4}\right)$, and $1\left(3 \% \mathrm{PH}_{3}\right.$ in $\left.\mathrm{H}_{2}\right)$ sccm with a $\left[\mathrm{PH}_{3}\right]$ to $\left[\mathrm{PH}_{3}+\mathrm{H}_{2}+\mathrm{SiH}_{4}\right]$ gas ratio of $5000 \mathrm{ppm}$. Second, the n-type a-Si:H layer is patterned and etched by reactive-ion etching (RIE) to define the channel region of the TFT. Following that, the sample is sent to a furnace for $10 \mathrm{~min}$ at $500{ }^{\circ} \mathrm{C}$ under a nitrogen environment 
TABLE I

Device Dimensions of E1, E2, AND E3 PoLY-Si TFTs

\begin{tabular}{lccccc}
\hline $\begin{array}{l}\text { Device } \\
\text { Name }\end{array}$ & $\begin{array}{c}\text { Channel length } \\
(\boldsymbol{\mu m})\end{array}$ & $\begin{array}{c}\mathbf{W}_{\mathbf{1}} \\
(\mathbf{n m})\end{array}$ & $\begin{array}{c}\mathbf{W}_{\mathbf{2}} \\
\mathbf{( n m )}\end{array}$ & $\begin{array}{c}\text { Channel } \\
\text { Number }\end{array}$ & $\begin{array}{c}\text { Total Effective } \\
\text { Channel Width } \\
(\mathbf{n m})\end{array}$ \\
\hline E1 & 5 & 400 & 55.9 & 1 & 512 \\
\hline E2 & 5 & 200 & 57.7 & 2 & 631 \\
\hline E3 & 5 & 40 & 75.3 & 10 & 1905 \\
\hline
\end{tabular}

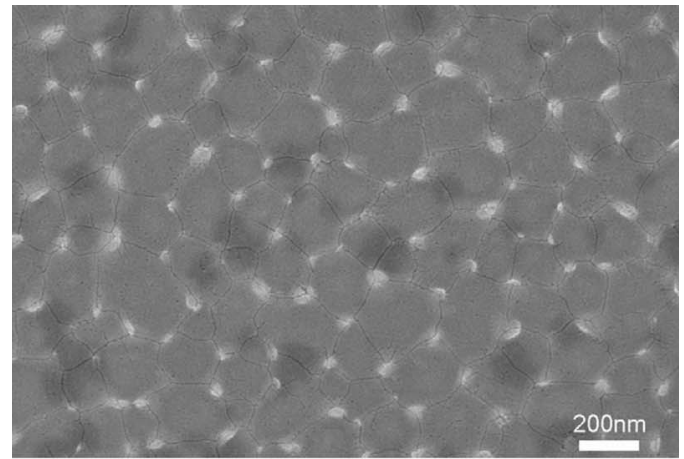

(a)

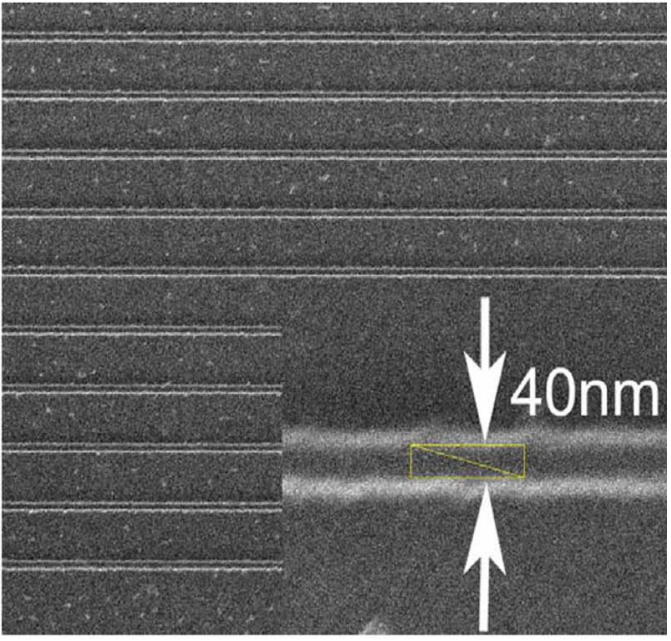

(b)

Fig. 2. (a) SEM photograph of poly-Si grain prepared $\mathrm{KrF}$ excimer laser annealing after Secco etching. The average grain size is $200 \mathrm{~nm}$. (b) SEM image of the E3 device at the channel region. The inset is an enlargement of one 40-nm-wide channel.

in order to expel hydrogen. After irradiating by excimer laser ( $\mathrm{KrF}$, the pulse duration time is $28 \mathrm{~ns}, 330 \mathrm{~mJ} / \mathrm{cm}^{2}$ ) at room temperature, multiple nanoscaled channels are patterned by electron beam lithography and transferred by RIE. A 100-nmthick $\mathrm{SiO}_{x}$ is subsequently deposited by PECVD at $250{ }^{\circ} \mathrm{C}$ as a gate insulator. After opening the contact windows for source and drain, the sample is treated by $\mathrm{NH}_{3}$ plasma at $250{ }^{\circ} \mathrm{C}$ for $3 \mathrm{~h}$, with a pressure of 2 torr and an RF power density of $0.44 \mathrm{~W} / \mathrm{cm}^{2}$. Finally, $150-\mathrm{nm}$-thick aluminum is evaporated on the devices to form ohmic contact and gate/source/drain electrodes. The postmetal annealing condition is $5 \mathrm{~min}$ at $400{ }^{\circ} \mathrm{C}$ under a nitrogen environment.

The current-voltage $(I-V)$ characteristics of fabricated devices are measured by an HP 4156 semiconductor parameter analyzer.

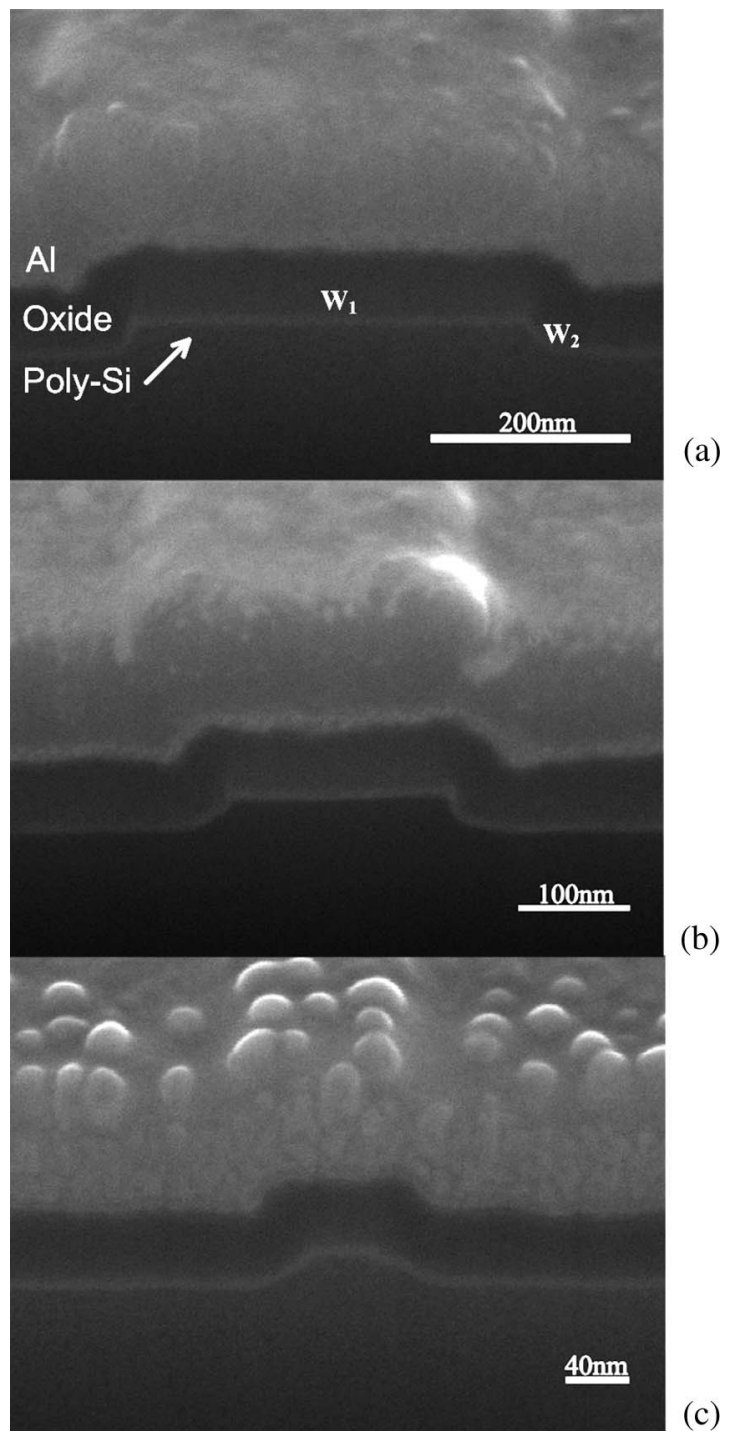

Fig. 3. Cross-sectional SEM image of the (a) E1, (b) E2, and (c) E3 devices along the $A A^{\prime}$ direction in Fig. 1.

\section{RESULTS AND DISCUSSION}

In this paper, TFTs are divided into three groups based on their channel number and width, i.e., 400-nm single channel (E1), 200-nm dual channel (E2), and ten 40-nm multichannel (E3), which are with the same channel length $(5 \mu \mathrm{m})$, as listed in Table I.

Fig. 2(a) and (b) shows the SEM image of the poly-Si channel layer before and after patterning the channel. In Fig. 2(a), after excimer laser annealing and Secco etching, the poly-Si grains with very clear boundaries are observed. The average grain size is about $200 \mathrm{~nm}$. Fig. 2(b) shows the SEM image of the channel region of the E3 device. Each channel in the E3 device is $40 \mathrm{~nm}$ wide, and the distance between each neighboring channel is $1 \mu \mathrm{m}$. The inset of Fig. 2(b) is the enlargement of one 40-nm-wide poly-Si channel.

Fig. 3(a)-(c) shows, respectively, the cross-sectional SEM images of the E1, E2, and E3 devices along the $A A^{\prime}$ direction in Fig. 1. It is obvious that the poly-Si channel region is trapezoidal in shape. Moreover, due to the 100-nm-thick $\mathrm{SiO}_{x}$, 


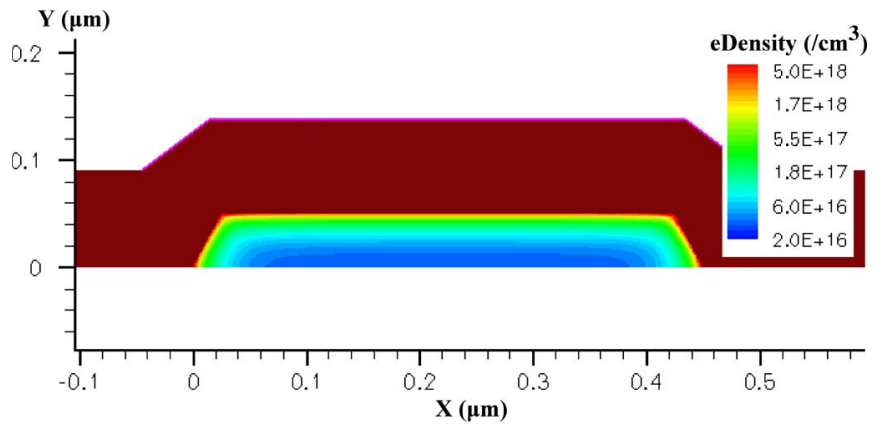

(a)

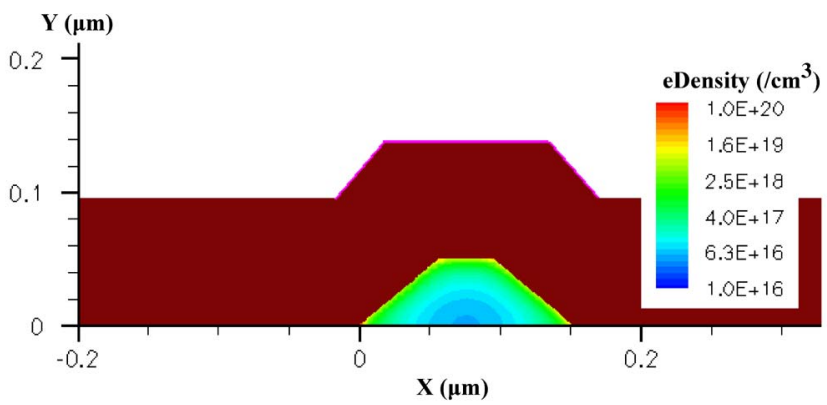

(b)

Fig. 4. Electron density distribution of the (a) E1 and (b) E3 devices. The voltage applied in the simulation is $V_{\mathrm{GS}}=5 \mathrm{~V}$ and $V_{\mathrm{DS}}=0.1 \mathrm{~V}$.

the cross section of these devices are different from that of the double gate FinFETs [10].

Fig. 4(a) and (b) shows the electron density distribution of the E1 and E3 devices by using the TCAD simulator. The construction of the simulation models is based on the crosssectional SEM images in Fig. 3(a) and (c). The trap model of the grain boundary in our simulation is based on the method of Levinson et al. [11]. Considering that the poly-Si grain size is about $200 \mathrm{~nm}$, it is assumed that there is one grain boundary in E1 (the channel width is twice that of the grain size) and there is no grain boundary in E3 (the channel width is smaller than the grain size) along the $A A^{\prime}$ direction. Apparently, the electron density on the top side $\left(W_{1}\right)$ of the channel is very much the same as that on the two sidewalls $\left(W_{2}\right)$. Hence, these two devices are effectively under trigate control. The sidewall conduction width $\left(W_{2}\right)$ in Fig. 3, then, must be counted into the total effective channel width. Accordingly, the total effective channel width is $\left(W_{1}+2 W_{2}\right)$ times the channel number. As a result, the effective channel widths of E1, E2, and E3 devices are 512, 631, and $1905 \mathrm{~nm}$, respectively.

In order to examine the effect of multichannel and narrow channel widths, the electrical characteristics of the three kinds of TFTs are compared. Fig. 5 shows the normalized transfer curve of the E1, E2, and E3 devices. It is clear that the on current of the $\mathrm{E} 3$ device at the gate bias $V_{\mathrm{GS}}=5.3 \mathrm{~V}$ and the drain bias $V_{\mathrm{DS}}=1 \mathrm{~V}$ is approximately $20 \%-25 \%$ higher than those of the other two devices. Additionally, the leakage current of the E3 device is reduced about two orders of magnitude compared with those of the E1 and E2 devices. Therefore, the E3 devices have a higher on/off current ratio $\left(>10^{7}\right)$ than the others.

Fig. 6 shows the normalized output characteristics of the E1, $\mathrm{E} 2$, and $\mathrm{E} 3$ devices. The applied gate voltage is $0-4 \mathrm{~V}$. The drain

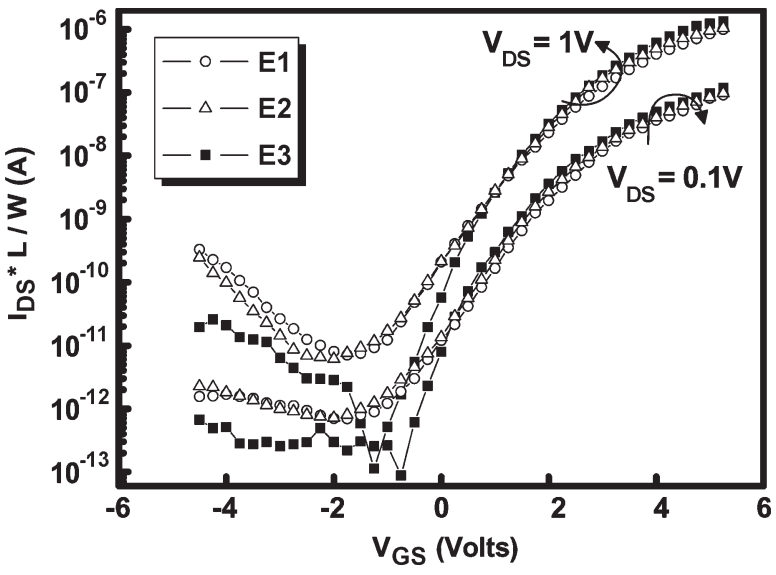

Fig. 5. Normalized transfer curves of LTPS TFTs with different width to length ratios $(W / L=512 / 5,631 / 5$, and $1905 / 5 \mathrm{~nm} / \mu \mathrm{m})$.

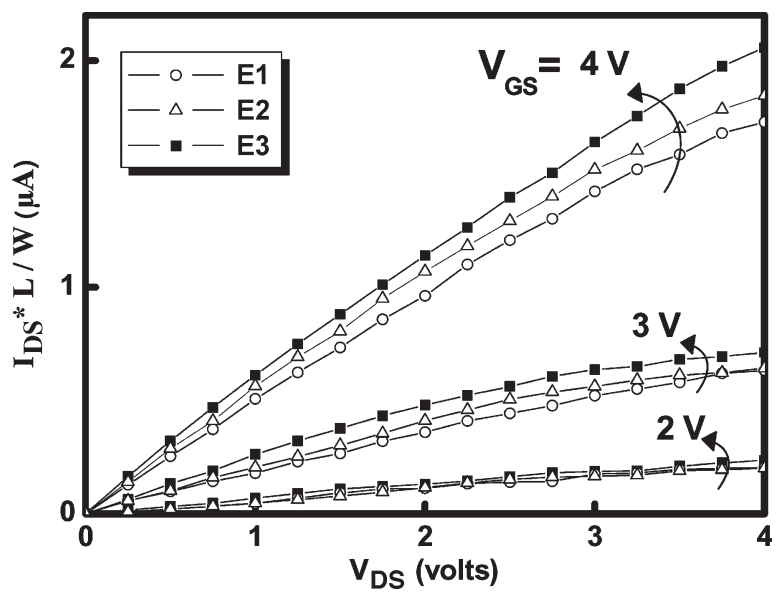

Fig. 6. Comparison of normalized $I_{D}-V_{D}$ curve of LTPS TFTs with different width to length ratios $(W / L=512 / 5,631 / 5$, and $1905 / 5 \mathrm{~nm} / \mu \mathrm{m})$.

current $I_{\mathrm{DS}}$ of the E3 device at the gate bias $V_{\mathrm{GS}}=4 \mathrm{~V}$ and drain bias $V_{\mathrm{DS}}=4 \mathrm{~V}$ is $16 \%$ higher than that of the $\mathrm{E} 1$ device and $10 \%$ higher than that of the $\mathrm{E} 2$ devices.

Fig. 7 shows the plots of (left) the TFTs' minimum leakage current and the (right) maximum on/off current ratio of the E1, $\mathrm{E} 2$, and $\mathrm{E} 3$ devices. The minimum leakage current is defined as the minimum drain current $I_{\mathrm{DS}}$ at the drain bias $V_{\mathrm{DS}}=0.1 \mathrm{~V}$. The maximum on/off current ratio is defined as $V_{\mathrm{DS}}=1 \mathrm{~V}$. As can be seen, the leakage current is almost the same for both E1 and E2 devices. However, the minimum leakage current of E3 decreases to one order of magnitude compared with the other two devices. It is because the E3 TFTs have a multichannel structure, which results in a larger surface area passivated by $\mathrm{NH}_{3}$ plasma. In addition, the plasma treatment can further reduce the number of grain boundary defects and lead to low leakage current [12], [13]. Moreover, the maximum on/off current ratio rises with the increase of the channel number. It can be explained that the E3 TFT structure has a better trigate control capability.

The statistical distributions of the threshold voltage and field effect mobility for three different devices are shown in Fig. 8. The mobility is obtained from the maximum transconductance at a low drain voltage $V_{\mathrm{DS}}=0.1 \mathrm{~V}$. The threshold voltage is 


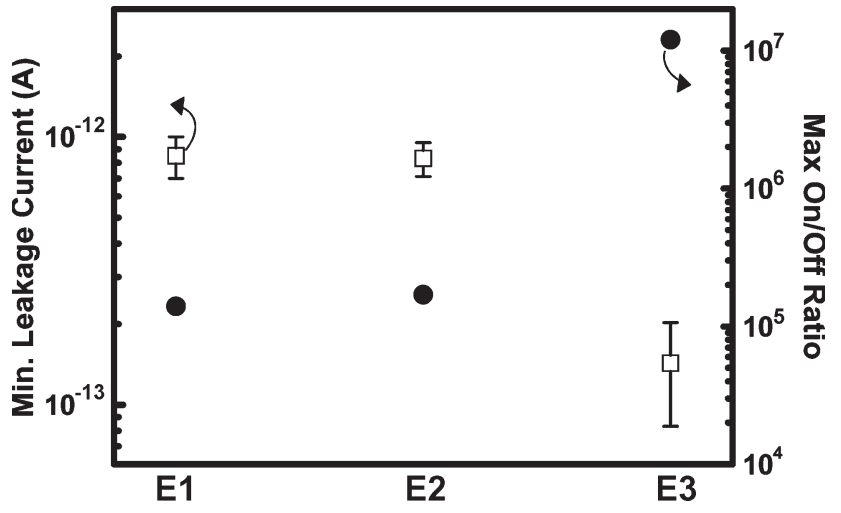

Fig. 7. Minimum leakage current and maximum on/off current ratio of the $\mathrm{E} 1, \mathrm{E} 2$, and E3 devices.

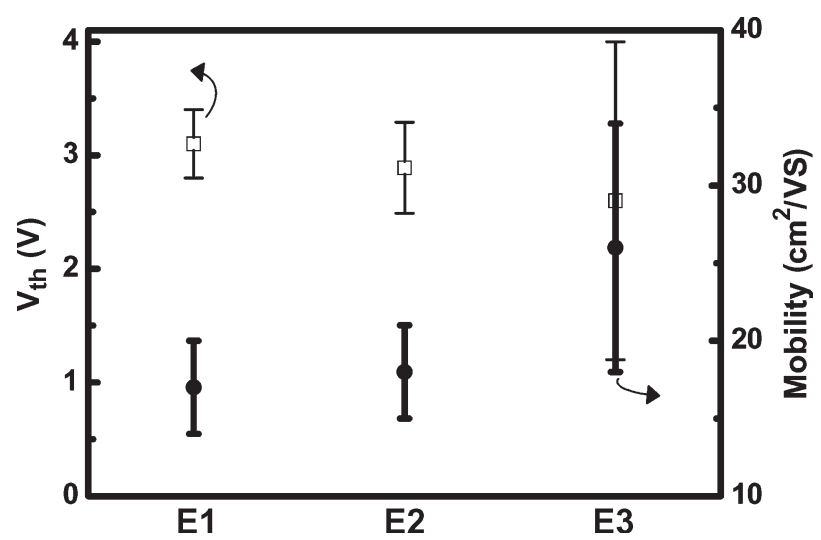

Fig. 8. Statistics of threshold voltage $\left(V_{\mathrm{th}}\right)$ and field effect mobility $(\mu)$ of the $\mathrm{E} 1, \mathrm{E} 2$, and $\mathrm{E} 3$ devices.

defined as the gate bias for a normalized drain current of $I_{\mathrm{DS}} \times$ $L / W=10^{-8} \mathrm{~A}$ at $V_{\mathrm{DS}}=0.1 \mathrm{~V}$. The threshold voltage of three poly-Si TFTs with a single channel (E1) and double (E2) and ten $(\mathrm{E} 3)$ channels fabricated by ELA are $3.1( \pm 0.3), 2.9( \pm 0.4)$, and $2.6( \pm 1.4) \mathrm{V}$, respectively. Moreover, the mobility of three poly-Si TFTs with a single channel (E1) and double (E2) and ten (E3) channels fabricated by ELA are $17( \pm 3), 18( \pm 3)$, and $26( \pm 8) \mathrm{cm}^{2} / \mathrm{V} \cdot \mathrm{s}$, respectively. Clearly, the variations of the $V_{\text {th }}$ and mobility increase with the increasing channel number and decreasing channel dimension. The explanation may be that when the channel width is smaller than the grain size of the poly-Si, the number of grain boundaries in the channel is not uniform, which will affect the uniformity of the electrical characteristics [9], [14].

To investigate the effect of grain boundaries in the three kinds of LTPS TFTs, the grain boundary trap-state densities $\left(N_{\text {trap }}\right)$ are estimated by the method of Levinson et al. [11]. The relationship between $I_{\mathrm{DS}}$ and $V_{\mathrm{DS}}$ is written as follows in the linear region:

$$
\begin{aligned}
I_{\mathrm{DS}}=(W / L) C_{\mathrm{OX}} V_{\mathrm{DS}} V_{G} & \\
& \times \exp \left(-q^{3} N_{\text {trap }}^{2} d / 8 K T \varepsilon_{S} C_{\mathrm{OX}} V_{G}\right)
\end{aligned}
$$

where $C_{\mathrm{OX}}$ is the gate oxide capacitance per unit area, $\mu_{g}$ is the carrier mobility at the $\mathrm{Si}$ grain boundary, $\varepsilon_{S}$ is the dielectric

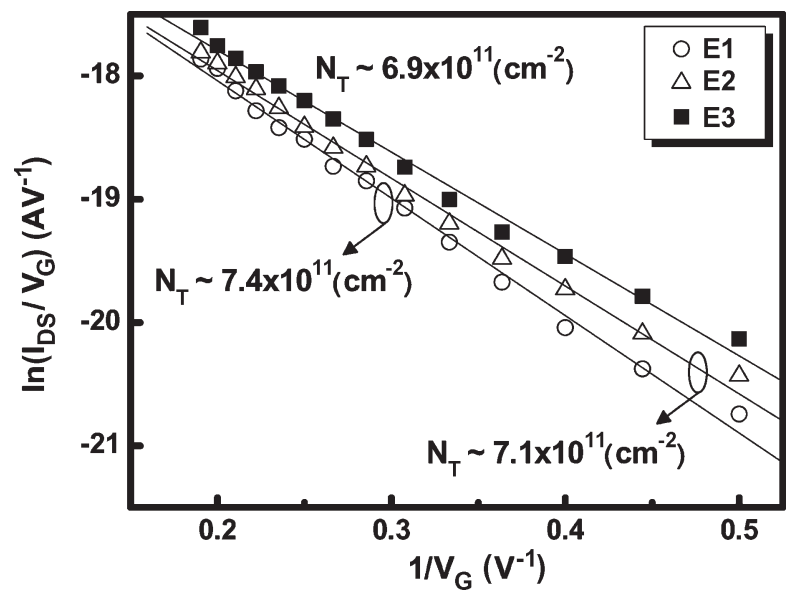

Fig. 9. Plot of $\ln \left(I_{\mathrm{DS}} / V_{G}\right)$ versus $\left(1 / V_{G}\right)$ for E1, E2, and E3 low temperature poly-Si TFTs.

constant for $\mathrm{Si}$, and $d$ is the thickness of the induced channel in the poly-Si.

Therefore, the $N_{\text {trap }}$ is extracted from the slopes of $\ln \left(I_{\mathrm{DS}} / V_{G}\right)$ versus $1 / V_{G}$ at $V_{\mathrm{DS}}=0.1 \mathrm{~V}$ and high $V_{G}$. It is shown in Fig. 9 that the E3 device exhibits an $N_{\text {trap }}$ of $6.9 \times 10^{11} / \mathrm{cm}^{2}$, which is 1.1 times smaller than that of the E1 and E2 devices, i.e., $\sim 7.4 \times 10^{11} / \mathrm{cm}^{2}$. This result implies that the E3 TFTs, with ten 40-nm-wide channels, posses fewer grain boundary defects density, which are also supported by the lower $V_{\text {th }}$ and less leakage current.

\section{CONCLUSION}

In conclusion, the LTPS TFTs prepared by $\mathrm{KrF}$ excimer laser annealing with multiple channels have been successfully fabricated. The trigate structure is confirmed by crosssectional SEM. The TFTs with ten channels exhibit better electrical performance than those with single and dual channels, i.e., a higher on/off current ratio $\left(>10^{7}\right)$, lower leakage current $\left(8.8 \times 10^{-14} \mathrm{~A}\right)$, minimum grain boundary defects density $\left(6.9 \times 10^{11} \mathrm{~cm}^{-2}\right)$, and a better subthreshold swing $(0.45 \mathrm{~V} / \mathrm{dec})$. It is found that when the channel width is smaller than the poly-Si grain size, the uniformity of the mobility and threshold voltage degrades.

\section{REFERENCES}

[1] S. Inoue, S. Utsunomiya, T. Saeki, and T. Shimoda, "Surface-free technology by laser annealing (SUFTLA) and its application to poly-Si TFTLCDs on plastic film with integrated drivers," IEEE Trans. Electron Devices, vol. 49, no. 8, pp. 1353-1360, Aug. 2002.

[2] C. C. Wu, S. D. Theiss, G. Gu, M. H. Lu, J. C. Sturm, S. Wagner, and S. R. Forrest, "Integration of organic LEDs and amorphous Si TFTs onto flexible and lightweight metal foil substrates," IEEE Electron Device Lett., vol. 18, no. 12, pp. 609-612, Dec. 1997.

[3] T. Serikawa, S. Shirai, A. Okamoto, and S. Suyama, "Low-temperature fabrication of high-mobility poly-Si TFTs for large-area LCDs," IEEE Trans. Electron Devices, vol. 36, no. 9, pp. 1929-1933, Sep. 1989.

[4] Y. Morimoto, Y. Jinno, K. Hirai, H. Ogata, T. Yamada, and K. Yoneda, "Influence of the grain boundaries and intragrain defects on the performance of poly-Si thin film transistors," J. Electrochem. Soc., vol. 144, no. 7, pp. 2495-2501, Jul. 1997.

[5] I. H. Song, C. H. Kim, S. H. Kang, W. J. Nam, and M. K. Han, "A new multi-channel dual-gate poly-Si TFT employing excimer laser annealing recrystallization on pre-patterned a-Si thin film," in IEDM Tech. Dig., 2002, pp. 561-564. 
[6] Y.-C. Wu, T.-C. Chang, C.-Y. Chang, C.-S. Chen, C.-H. Tu, P.-T. Liu, H.-W. Zan, and Y.-H. Tai, "High-performance polycrystalline silicon thinfilm transistor with multiple nanowire channels and lightly doped drain structure," Appl. Phys. Lett., vol. 84, no. 19, pp. 3822-3824, May 2004.

[7] T. Unagami and O. Kogure, "Large on/off current ratio and low leakage current poly-Si TFTs with multichannel structure," IEEE Trans. Electron Devices, vol. 35, no. 11, pp. 1986-1989, Nov. 1988.

[8] H.-W. Zan, T.-C. Chang, P.-S. Shih, D.-Z. Peng, T.-Y. Huang, and C.-Y. Chang, "Analysis of narrow width effects in polycrystalline silicon thin film transistors," Jpn. J. Appl. Phys., vol. 42, no. 1, pp. 28-32, 2003.

[9] N. Yamauchi, J.-J. J. Hajjar, and R. Reif, "Polysilicon thin-film transistors with channel length and width comparable to or smaller than the grain size of the thin film," IEEE Trans. Electron Devices, vol. 38, no. 1, pp. 55-60, Jan. 1991.

[10] B. Yu, L. Chang, S. Ahmed, H. Wang, S. Bell, C.-Y. Yang, C. Tabery, C. Ho, Q. Xiang, T.-J. King, J. Bokor, C. Hu, M.-R. Lin, and D. Kyser, "FinFET scaling to $10 \mathrm{~nm}$ gate length," in IEDM Tech. Dig., 2002, pp. 251-254.

[11] J. Levinson, F. R. Shepherd, P. J. Scanlon, W. D. Westwood, G. Este, and M. Rider, "Conductivity behavior in polycrystalline semiconductor thin film transistors," J. Appl. Phys., vol. 53, no. 2, pp. 1193-1202, Feb. 1982.

[12] Y.-C. Wu, T.-C. Chang, C.-W. Chou, Y.-C. Wu, P.-T. Liu, C.-H. Tu, W.-J. Huang, J.-C. Lou, and C.-Y. Chang, "Effects of channel width and $\mathrm{NH}_{3}$ plasma passivation on electrical characteristics of polysilicon thinfilm transistors by pattern-dependent metal-induced lateral crystallization," J. Electrochem. Soc., vol. 152, no. 7, pp. G545-G549, 2005.

[13] H.-C. Cheng, F.-S. Wang, and C.-Y. Huang, "Effects of $\mathrm{NH}_{3}$ plasma passivation on N-channel polycrystalline silicon thin-film transistors," IEEE Trans. Electron Devices, vol. 44, no. 1, pp. 64-68, Jan. 1997.

[14] T. Noguchi, A. J. Tang, J. A. Tsai, and R. Reif, "Comparison of effects between large-area-beam ELA and SPC on TFT characteristics," IEEE Trans. Electron Devices, vol. 43, no. 9, pp. 1454-1458, Sep. 1996.

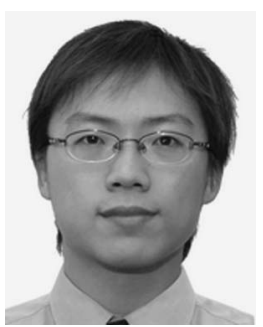

Po-Chuan Yang was born in Taiwan, R.O.C., on January 3, 1981. He received the B.S. degree in mechanical engineering from National Taiwan University (NTU), Taipei, Taiwan, in 2003.

$\mathrm{He}$ is currently with the Graduate Institute of Electronics Engineering, NTU. His research interests are low-temperature polycrystalline silicon thin-film transistors and flexible electronics.

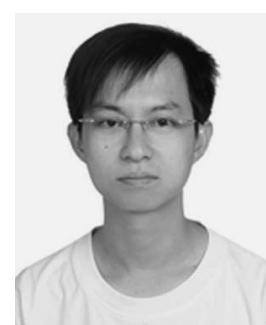

Ping-Sheng Kuo was born in Taiwan, R.O.C., on January 29,1978 . He received the B.S. degree in electric engineering from National Cheng Kung University, Tainan, Taiwan, in 2002 and the M.S. degree in the electric engineering from National Taiwan University (NTU), Taipei, Taiwan, in 2004.

$\mathrm{He}$ is currently with the Graduate Institute of Electronics Engineering, NTU. His research interests are the transport mechanism of SiGe-quantum dot devices and FinFET devices.

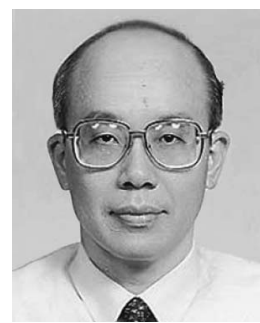

Si-Chen Lee (A'84-SM'90-F'02) was born in Taiwan, R.O.C., on August 13, 1952. He received the B.S. degree in electrical engineering from National Taiwan University (NTU), Taipei, Taiwan, in 1974 and the Ph.D. degree in electrical engineering from Stanford University, Stanford, CA, in 1981, with a work consisting of the experimental investigation of $\mathrm{AlGaAs} / \mathrm{GaAs}$ multiheterojunction properties.

From 1980 to 1982 , he was with Energy Conversion Devices Inc., Troy, MI, where his work was concerned with the application of amorphous silicon hydrogen alloy to solar cells. Since 1982, he has been with NTU, where he was an Associate Professor with the Department of Electrical Engineering, the Department Chairman from 1988 to 1992, and the Dean of Academic Affairs from 1996 to 2002; currently, he is with the Department of Electrical Engineering, where he is a Professor, and with the Graduate Institute of Electronics Engineering. Since 2005, he has been the President of NTU. His current research interests are in the device applications and the growth kinetics of InGaAs/GaAs strained layer quantum dot device, InGaAs/InAs room temperature infrared light emitting diode and photodetector with applications to pollution detection and the biological reaction of cells. In addition, he is also interested in hydrogenated and deuterated amorphous and poly-Si (carbon, germanium) hydrogen material and devices, such as thin-film transistors and neural network image sensors. Since 1988, he pioneered a research work on the Chinese traditional qigong and somatic science.

Dr. Lee is a member of the Chinese Institute of Electrical Engineering. He has received Dr. Sun Yat-San Academic Award in 1987 and five consecutive outstanding research awards of the National Science Council from 1986 to 1996. He has been elected as a member of The Asia-Pacific Academy of Materials in 1997, and received the IEEE Third Millennium Medal for outstanding achievements and contributions in the area of semiconductor devices in 2000. In 2002, he was awarded the Medal of Electrical Engineering from the Association of Chinese Electrical Engineers. He has received the 47th Academic Award of the Ministry of Education in 2003. 